\title{
Solving acoustic scattering problem by the meshless method based on radial basis function
}

\author{
Weifang Liu $^{1, *}$, Sanfei Zhao ${ }^{1}$, Cong Zhang $^{1}$ and Huipo Qiao ${ }^{1}$ \\ ${ }^{1}$ Marine engineering, China Ship Development and Design Center, China
}

\begin{abstract}
In this paper, radial point collocation method (RPCM) is introduced to solve the acoustic scattering problem. This is a mathematically simple, easy-to-program and truly meshless method, which has been successfully applied to solve the solid mechanics and convection diffusion problems. However, application of this method to investigate acoustic problems, in particle the acoustic scattering problem is relatively new. The main advantage of this method is its mathematically simple, easy to program, and truly meshless. A Hermite-type interpolation method is employed to improve the solution accuracy while the Neumann boundary conditions exist. In addition, acoustic scattering problem is a typical unbounded domain problem, in order to solve it with RPCM, the domain is truncated to a finite region and an artificial boundary condition (ABC) is imposed. Finally, numerical example is presented to validate the accuracy and effectiveness of RPCM. In the future, the extension of RPCM to more complex and practical problems, especially the three-dimensional situations need to be investigated in more detail.
\end{abstract}

\section{Introduction}

Historically, the acoustic scattering problem has been investigated quite extensively since the works of Lamb and Rayleigh ${ }^{[1]}$. This kind of problem can be described as the solution of the Helmholtz equation in an unbounded domain. A profusion of numerical approaches have been developed to solve this problem. Among these methods, finite element method (FEM) and boundary element method (BEM) are widely used.

However, both FEM and BEM need mesh generation in the preparation of data, and the BEM has to face the singular and hypersingular integrals. Furthermore, it is proved that for high wave numbers, the FEM has the pollution effect which leads to inaccurate results. To avert these difficulties, meshless methods, as relatively new methods, have attracted researchers' attention ${ }^{[2-6]}$.

RPCM is a meshless method based on the combination of radial basis (RBF) and collocation scheme. Since RBF is used to formulate the shape function for RPCM, there are generally little restrictions on the way the data prescribed and the method has the applicability in almost any dimension. In addition, problem is discretized by the collocation scheme directly, therefore, no integrations is needed. And it has been successfully used to solve the solid mechanics and convection diffusion problems ${ }^{[7]}$. However, applications concerning acoustic problems, especially an investigation of the acoustic scattering problems are relatively new.

The goal of this paper is to apply RPCM to solve acoustic scattering problem. For Neumann boundary conditions, Hermite interpolation is applied to handle them. Considering that RPCM can not approach the exterior problems directly, absorbing boundary condition is introduced. To validate the effectiveness and accuracy of the proposed method for the acoustic scattering problem, numerical example is presented.

The outline of the paper is as follows: RPCM is described briefly in Section 2. In Section 3, acoustic scattering problem is described. The acoustic scattering problem solved with RPCM are presented in Section 4. Finally, Section 5 draws the conclusion.

\section{Outline of the RPCM}

\subsection{Hermite-type radial basis interpolation}

Until now, various RBFs have been proposed, and the MQ is widely used for its efficiency.

$$
R_{i}(x, y)=\left(r_{i}^{2}+(c d)^{2}\right)^{q}, c \geq 0, q_{(\mathrm{MQ})}
$$

Where $\mathrm{c}$ is the shape parameter, $r_{i}$ is distance between the point of interest and the supported interpolation points, $\mathrm{d}$ is a characteristic length.

Considering a continuous function $\mathrm{u}(\mathrm{x})$ defined in a domain, the $\mathrm{u}(\mathrm{x})$ at the point of interest $\mathrm{x}$ can be approximated with radial basis interpolaion as follows:

$$
u^{h}(\mathbf{x})=\sum_{i=1}^{n} R_{i}(\mathbf{x}) a_{i}+\sum_{j=1}^{n_{D B}} \frac{\partial R_{j}^{D B}(\mathbf{x})}{\partial \mathrm{n}} b_{j}+\sum_{k=1}^{m} p_{k}(\mathbf{x}) c_{k}
$$

\footnotetext{
Corresponding author: 5543385@qq.com
} 
Where $\mathrm{R}_{\mathrm{i}}(\mathrm{x})$ and $\mathrm{R}_{\mathrm{j}}^{\mathrm{DB}}(\mathrm{x})$ are both MQ RBF, $\mathrm{n}$ is the number of the RBF, $m$ is the number of polynomial basis function. $\mathrm{n}_{\mathrm{DB}}$ is the number of the points on the Numman boundary conditions.

\subsection{Collocation scheme}

Meshless methods can be divided into three categories according the formulation procedures of the governing equations: meshless methods based on weak-forms, meshless methods based on strong-forms (collocation techniques), meshless methods based on the combination of the weak-form and the strong form. In RPCM, the formulation procedure of discretization equations is the second one. With this method, the partial differential equation (PDF) is generally discretized at nodes by some forms of collocation. For the sake of simplicity, we consider the following boundary value problem:

$$
\operatorname{Lu}(x)=f(x), x \in \Omega
$$

together with the Dirichlet boundary :

$$
B u(x)=g(x), x \in \Gamma .
$$

Where $\mathrm{L}$ is a partial differential operator and the $\mathrm{B}$ is a boundary operator, $\mathrm{f}(\mathrm{x})$ and $\mathrm{g}(\mathrm{x})$ are known function. Assume that there are totally points for discretization, $n_{i}$ interior points and $\mathrm{n}_{\mathrm{b}}$ boundary points. By substituting Eq.(2) into Eq.(3) and Eq.(4), the following discrete equations can be obtained:

$$
\begin{aligned}
& \sum_{i=1}^{n} L\left(R_{i}\left(\mathbf{x}_{l}\right)\right) a_{i}+\sum_{j=1}^{n_{D B}} L\left(\frac{\partial R_{j}^{D B}\left(\mathbf{x}_{l}\right)}{\partial \mathrm{n}}\right) b_{j} \\
& +\sum_{k=1}^{m} L\left(p_{k}\left(\mathbf{x}_{l}\right)\right) c_{k}=f\left(\mathbf{x}_{l}\right) \quad l=1, \mathrm{~L} n_{i} . \\
& \sum_{i=1}^{n} B\left(R_{i}\left(\mathbf{x}_{l}\right)\right) a_{i}+\sum_{j=1}^{n_{D B}} B\left(\frac{\partial R_{j}^{D B}\left(\mathbf{x}_{l}\right)}{\partial \mathrm{n}}\right) b_{j} \\
& +\sum_{k=1}^{m} B\left(p_{k}\left(\mathbf{x}_{l}\right)\right) c_{k}=g\left(\mathbf{x}_{l}\right) \quad l=1 \mathrm{~L} n_{b} .
\end{aligned}
$$

Solving Eq.(5) and Eq.(6), the constant coefficients $a_{i}, b_{j}$ and $c_{k}$ can be obtained, thus the value of any point can be gotten through Eq.(2).

\section{Acoustic Scattering Problems}

Fig.1 shows a typical acoustic scattering problem, in which an incident wave $\mathrm{p}^{\mathrm{i}}$ scattered by an obstacle $V^{\prime}$ in an unbounded domain $V$. Considering $V$ is homogenous anisotropic medium with the state variables: density $\rho$, sound speed $\mathrm{c}$. The incident wave $\mathrm{p}^{\mathrm{i}}$ and scattering wave $\mathrm{p}^{\mathrm{s}}$ are governed by the following equation:

$$
\nabla^{2} P-\frac{1}{c^{2}} \frac{\partial^{2} P}{\partial t^{2}}=0
$$

Where $\nabla^{2}$ is the Laplace operator. If we suppose the wave is time harmonic with frequency $\omega$, the pressure can be presented as:

$$
P(x, t)=\operatorname{Re}\left(p(x) e^{-i \omega t}\right)
$$

Where $\mathrm{i}=\sqrt{-1}$, by introducing the wave number $k=\omega / c$ and velocity potential $\phi=-\frac{p}{i \omega \rho}$, the Helmholtz equation is obtained:

$$
\nabla^{2} \phi+k^{2} \phi=0
$$

As well known, the total sound pressure or velocity potential in the sound field can be divided into two parts:(1) incident wave field; (2) scattering wave field, which can be written as:

$$
\begin{aligned}
& p=p^{i}+p^{s} \\
& \phi=\phi^{i}+\phi^{s}
\end{aligned}
$$

Where $\phi^{i}$ and $\phi^{s}$ denote the incident velocity potential and the scattering velocity potential separately.

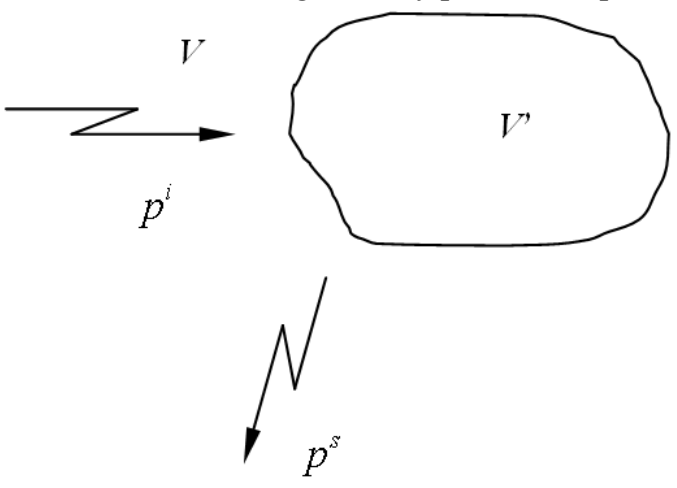

Fig. 1. Acoustic scattering problem

Different scattering obstacles have different boundary conditions on the surface of the obstacles, and there are generally three kinds of boundary conditions:

(1) A sound-hard object (Neumann boundary condition)

$$
\frac{\partial \phi}{\partial n}=0 \Leftrightarrow \frac{\partial \phi^{s}}{\partial n}=-\frac{\partial \phi^{i}}{\partial n}
$$

where $\mathrm{n}$ presents the vector normal to the surface of the object.

(2) A sound-soft object (Dirichlet boundary condition)

$$
\phi=0 \Leftrightarrow \phi^{i}+\phi^{s}=0
$$

(3) A convective obstacle (impedance, Robin boundary condition)

$$
\frac{\partial \phi}{\partial n}+i \lambda \phi=0
$$

where $\lambda \in C, \operatorname{Re}(\lambda) \geq 0$ stands for the impedance parameter. In order to guarantee that the scattering problem has a unique solution where the scattering wave is an outgoing wave, Sommerfeld radiation condition at infinity should be considered: 


$$
\lim _{r \rightarrow \infty} r^{(n-1) / 2}\left|\frac{\partial \phi^{s}}{\partial r}-i k \phi^{s}\right|=0
$$

Where $\mathrm{n}$ is the dimension of the problem.

\subsection{Treatments for unbounded domain problems}

In scattering theory, the acoustic scattering problem falls into the class of unbounded domain problems. In order to solving this kind of problems with the RPCM, special treatment is need. Among various treatments, absorbing boundary condition is an efficient numerical approach. We list the sequence boundary operators with different orders, developed by Bayliss, Turkel and Gunzburger ${ }^{[8]}$, as follows:

$$
\begin{gathered}
\frac{\partial u}{\partial r}-i k u=0 \\
\mathrm{BC} 0 \quad B_{1} u=\frac{\partial u}{\partial r}+\alpha u=0 \\
\mathrm{BC} 1 \quad \frac{1}{2 r}-i k \\
\mathrm{BC} 2 \quad B_{2} u=\frac{\partial^{2} u}{\partial r^{2}}+b_{1} \frac{\partial u}{\partial r}+b_{0} u=0 \\
b_{0}=-\frac{5}{4 r^{2}}-\frac{3 i k}{r}-k^{2} \\
b_{1}=\frac{3}{r}-2 i k
\end{gathered}
$$

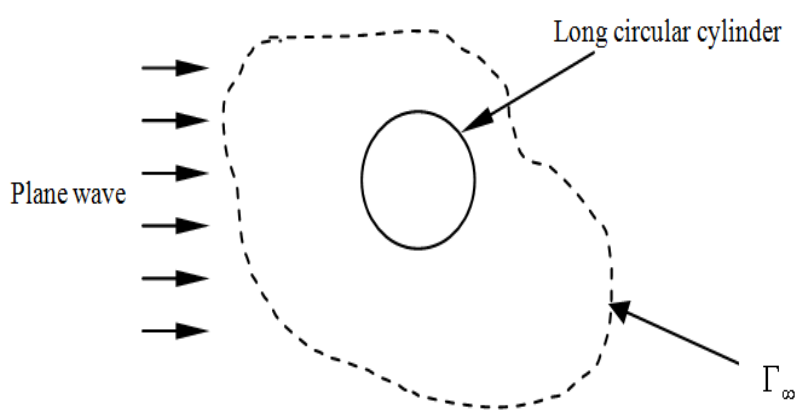

Fig. 2. Plane wave scattered by a rigid infinite circle cylinder

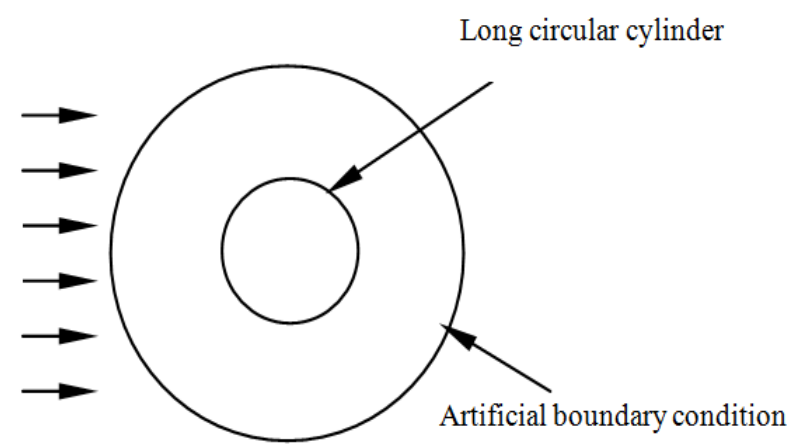

Fig. 3. Unbounded domain is truncated to a finite region with an artificial boundary condition

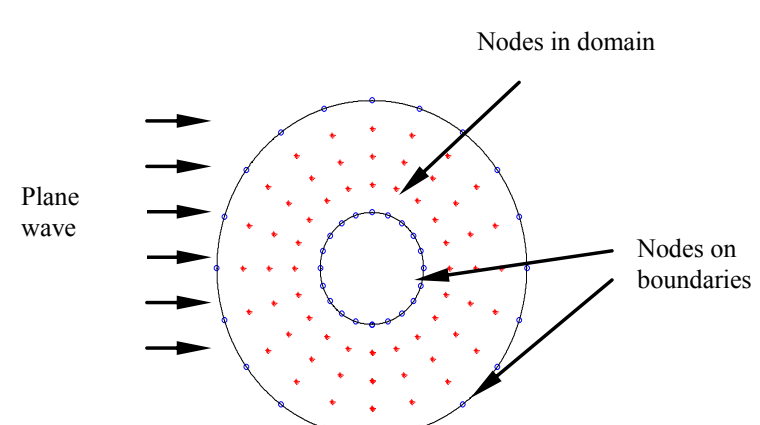

Fig. 4. Discretization of the solution domain and boundaries

\section{Numerical Example}

In this section, RPCM is used to solve the acoustic scattering problem shown in Fig.2. Considering a plane wave with unit amplitude scattered by a rigid infinite circle cylinder with radius a, for simplification, we investigate it in 2-dimension. It is a typical unbounded domain problem, in which the domain should be truncated to a finite region, shown as Fig.3.

Fig. 4 shows the discretization of the internal domain and the boundaries, a total of 40 uniform nodes are arranged on the boundary and 60 internal nodes are located in the domain.

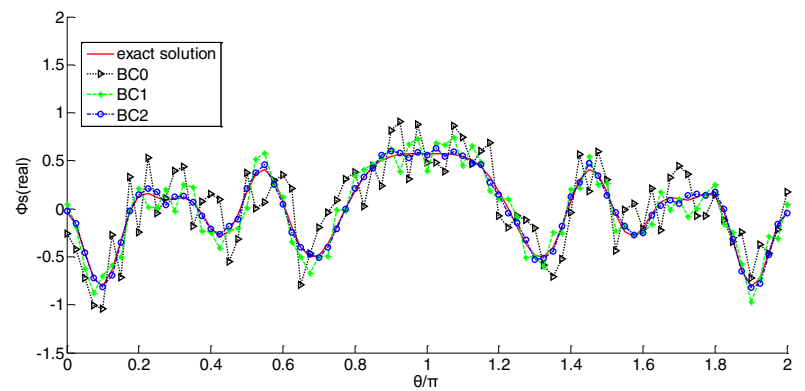

Fig. 5a. Plane wave scattered by a rigid cylinder when $k a=4 \pi, r=2 a$ ( $\mathrm{r}$ is the distance between the field point and the centre of the circle); real part of scattering wave

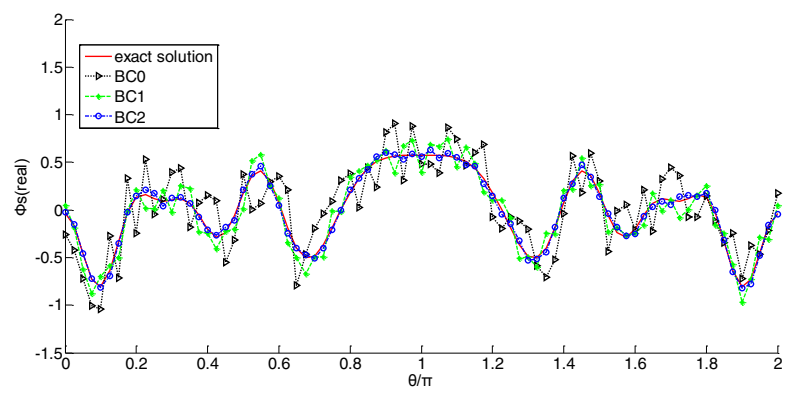

Fig. 5b. Plane wave scattered by a rigid cylinder when $\mathrm{ka}=4 \pi, \mathrm{r}=2 \mathrm{a}$ ( $\mathrm{r}$ is the distance between the filed point and the centre of the circle); image part of scattering wave.

Comparison between the exact solution and the numerical solutions with artificial boundary conditions are shown in Fig.5. We can Fig. out that artificial boundary condition plays an important role in the simulation. And it should be notied that, when the proper artificial boundary condition is chosen, the solution obtained with RPCM agrees very well with the exact solution. 


\section{Conclusions}

PRCM is a mathematically simple, easy-to-program and truly meshless method, which has been successfully applied to solve the solid mechanics and convection diffusion problems. However, application of this method to investigate acoustic problems, in particle the acoustic scattering problem is relatively new. In this paper, RPCM is introduced to approach the acoustic scattering problem, and the comparison between the numerical results with the exact solution demonstrates the effectiveness of this scheme. In the future, the extension of RPCM to more complex and practical problems, especially the three-dimensional situations need to be investigated in more detail.

\section{References}

1. Sir H. Lamb, Hydrodynamics, New York: Dover Publications; 1945.

2. Liu GR. Meshfree methods-moving beyond the finite element method. 2nd edition. Washington: CRC Press: 2009.
3. Wen Chen, Z.J. Fu, B.T. Jin. A truly boundary-only meshfree method for inhomogeneous problems based on recursive composite multiple reciprocity technique. Eng Anal Bound Elem 2010;34:196-205.

4. Wen Chen, Ji Lin, Fuzhang Wang. Regularized meshless method for nonhomogeneous problems. Eng Anal Bound Elem 2011;35:253-257.

5. K. li, Q.B.Huang, J.L. Wang, L.G. Lin. An improved localized radial basis function meshless method for computational aeroacoustics. Eng Anal Bound Elem 2011;35:47-55.

6. K. li, Q.B.Huang, Y. Miao. Dual reciprocity hybrid boundary node method for acoustic eigenvalue problems. Eng Anal Bound Elem 2010;34:359-368.

7. Liu GR. An Introduction to Meshfree Methods and Their Programming. Dordrecht,Netherlands:Springer; 2005.

8. Alvin Bayliss, Max Gunzburger, Eli Turkel. Boundary Conditions for the Numerical Solution of Elliptic Equations in Exterior Regions. Siam J Appl Math 1982;42:430-451. 